\title{
Exploring Again the Principle of Level Surveying
}

\author{
Yonghe Deng \\ Department of Civil Engineering, Lishui College, Lishui 323000,China \\ Isxydengyonghe@sina.com
}

Keywords: the principle of level surveying; level surface; horizontal surface; strict model;uncertainty

Abstract. Literature [1] studied the principle formulas of level surveying, but its theory is not strict enough. So, in this paper, further research is made to obtain more strict formulas, and proves that formula in literature [1] can completely replace homologous formula of this paper in practice.

\section{Introduction}

About principle formula of level surveying, Literature [1] is better than literatures[2-5] and literature[6], because it considers more completely and more reasonably. But in literature[1],because $A^{\prime} A / / B B^{\prime}$ exists nearly, does not strictly exist, figures of four sides such as $A^{\prime} A B_{0} B^{\prime}$ (in Fig.1), $A^{\prime} A_{0} B B^{\prime}$ (in Fig.2) and $A^{\prime} A_{0} B_{0} B^{\prime}$ (in Fig.3,Fig.4 and Fig.5) are not rectangle. So, based on characteristic of rectangle in literatrure[1], Eq.1,Eq.2,Eq.4,Eq. 6 and Eq. 8 are not strict enough. Then, we should put forward more strict formulas, and study if the difference between new formulas in this paper and formulas in literature [1] is too small to consider. These works are meaningful.

\section{Deducing new formulas and doing comparison research}

If $B B^{\prime}$ of Fig. 1 in literature[1] revolves around $B$ to $B B^{\prime}{ }_{0} / / A^{\prime} A$, and we suppose the horizontal sight through level, the horizontal surface and the level surface through $A$ intersect $B B_{0}^{\prime}$ at $B^{\prime}{ }_{0}, B_{00}$ and $B^{\prime \prime}{ }_{0}$ in order such as Fig.1,then,figure $A^{\prime} A B_{00} B^{\prime}{ }_{0}$ is rectangle. According to $B_{00} B^{\prime}{ }_{0}=A A^{\prime}$, we obtain $\left[h_{A B^{-}}-\frac{\left(A_{B_{0}}\right)^{2}}{2 R}+b\right] \cos i=a$ (There $\left.i=\frac{A_{B_{0}}}{R}\right)$, then

$$
h_{A B}=a \sec \frac{A_{B_{0}}}{R}+\frac{\left(A_{B_{0}}\right)^{2}}{2 R}-b
$$

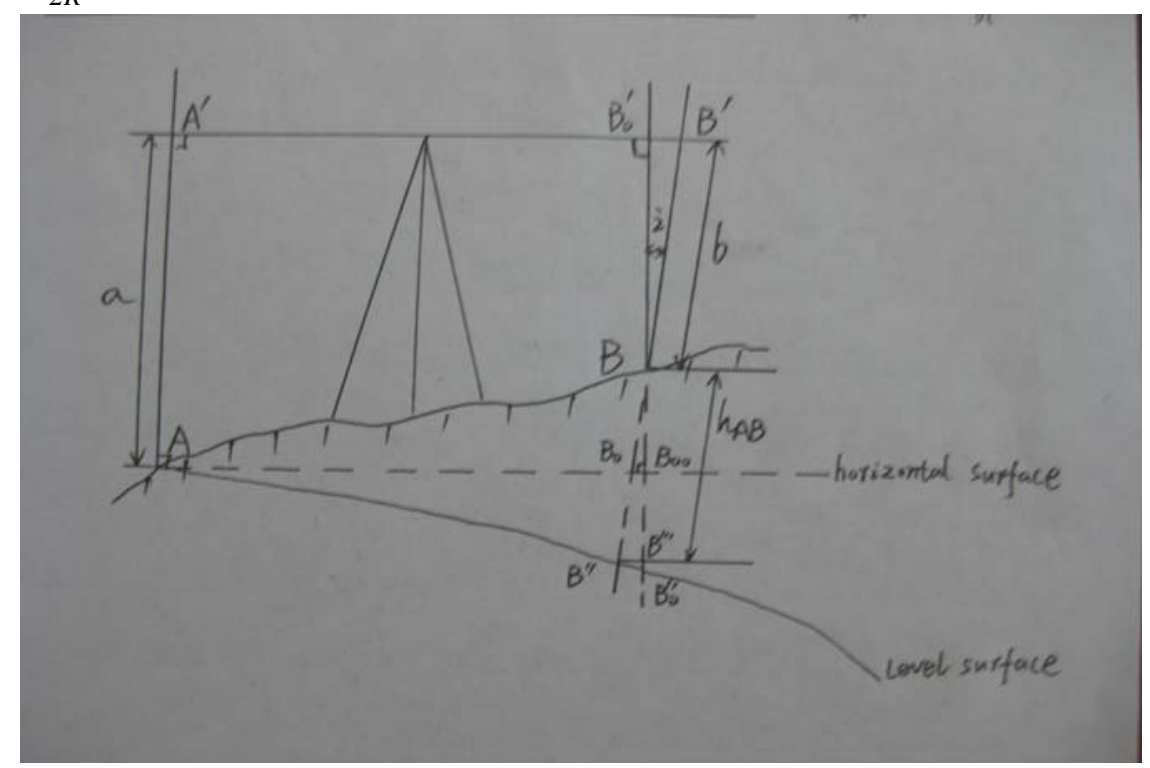

Fig 1 Selecting the horizontal surface and the level surface through point A to analyse Eq. 1 in this paper minus Eq.1 in literature[1] leaves

$\Delta h_{A B}=a\left(\sec \frac{A B_{0}}{R}-1\right)$ 
Based on Eq. 2 in this paper, because of $a\left\langle 3\right.$ meters, $A B_{0}\langle 200$ meters and $R=6370000$ meters in practice, we can get $\left|{ }_{h_{A B}}\right| 1.47 \times 10^{-6}$ millimeters. So, we can completely neglect the difference between Eq. 1 in literature[1] and Eq.1 in this paper.

If $A A^{\prime}$ of Fig.2 in literature[1] revolves around $\mathrm{A}$ to $A A^{\prime}{ }_{0} / / B B^{\prime}$, and we suppose the horizontal sight through level, the horizontal surface and the level surface through $B$ intersect $A A^{\prime}{ }_{0}$. at $A^{\prime}{ }_{0}, A_{00}$ and $A^{\prime \prime}{ }_{0}$ in order such as Fig.2, then,figure $A^{\prime}{ }_{0} A_{00} B B^{\prime}$ is rectangle. According to $A_{00} A^{\prime}{ }_{0}=B B^{\prime}$, we obtain $\left[a-h_{A B}-\frac{\left(B A_{0}\right)^{2}}{2 R}\right] \cos i=b\left(\right.$ There $\left.i=\frac{B A 0}{R}\right)$, then

$$
h_{A B}=a-b \sec \frac{B A_{0}}{R}-\frac{\left(B A_{0}\right)^{2}}{2 R}
$$

Eq.3 in this paper minus Eq.2 in literature[1] leaves

$$
\Delta h_{A B}=-b\left(\sec \frac{B A_{0}}{R}-1\right)
$$

Based on Eq. 4 in this paper, because of $b\left\langle 3\right.$ meters , $B A_{0}<200$ meters and $R=6370000$ meters in practice, we can get $\left|{ }_{h_{A B}}\right| 1.47 \times 10^{-6}$ millimeters. So, we can completely neglect the difference between Eq. 2 in literature[1] and Eq.3 in this paper.

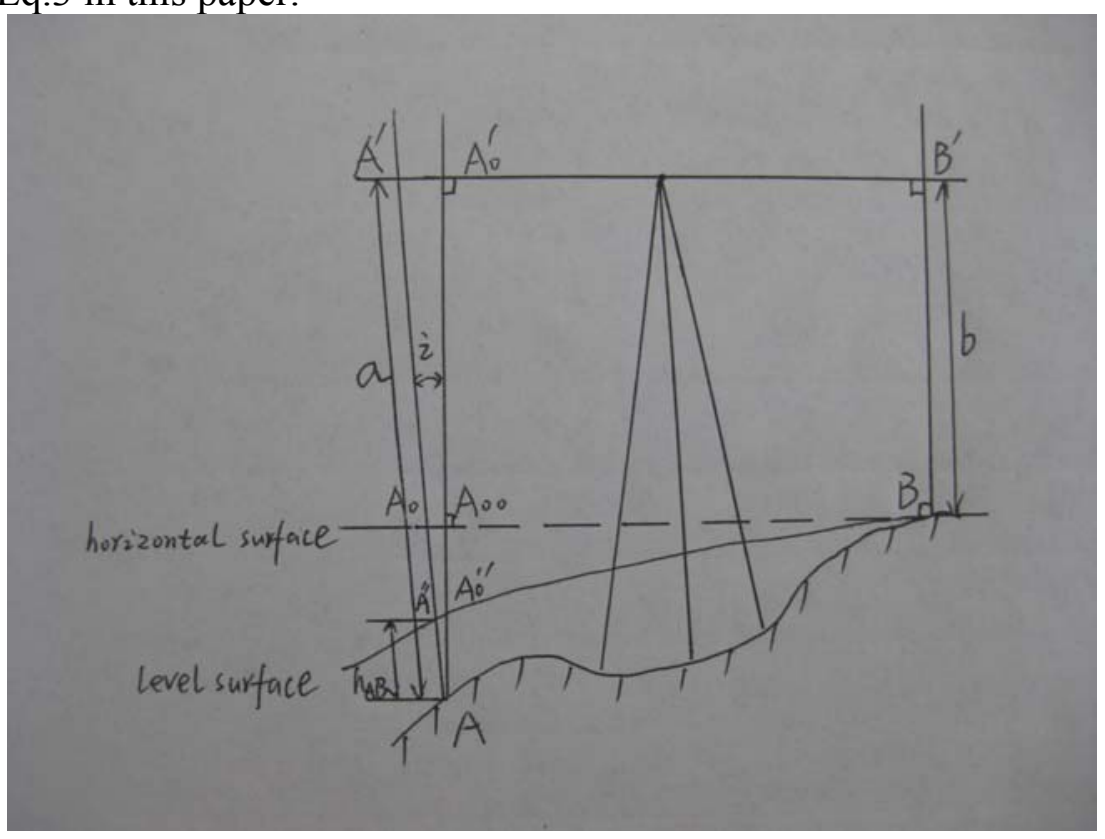

Fig 2 Selecting the horizontal surface and the level surface through point $B$ to analyse

If $B B^{\prime}$ of Fig.3 in literature[1] revolves around $B$ to ${ }^{B} B^{\prime}{ }_{0} \perp A_{0} B_{0}$, and $A A^{\prime}$ of Fig.3 in literature[1] revolves around $\mathrm{A}$ to $A A^{\prime}{ }_{0} \perp A_{0} B_{0}$, and we suppose the horizontal sight through level, the horizontal surface and the level surface through $C$ intersect $B B^{\prime}{ }_{0}$ and $A A^{\prime}{ }_{0}$ at $B_{0}^{\prime}, B_{00}, B^{\prime \prime}{ }_{0}$, and $A^{\prime}{ }_{0}, A_{00}, A^{\prime \prime}{ }_{0}$ in order such as Fig.3, then, figure $A_{0}^{\prime} A_{00} B_{00} B^{\prime}{ }_{0}$ is rectangle. According to $B_{00} B^{\prime}{ }_{0}=A_{00} A^{\prime}{ }_{0}$, we obtain $\left[a-h_{A C}-\frac{\left(A_{0} C\right)^{2}}{2 R}\right] \cos _{i_{1}}=\left[h_{C B}-\frac{\left(B_{0} C\right)^{2}}{2 R}+b\right] \cos i_{2},\left(\right.$ There $\left.i_{1}=\frac{C A_{0}}{R}, i_{2}=\frac{C_{B_{0}}}{R}\right)$, then

$$
h_{A B}=a+h_{C B}-\frac{\left(A_{0} C\right)^{2}}{2 R}+\left[\frac{\left(B_{0} C\right)^{2}}{2 R}-h_{C B}-b\right] \cos \frac{B_{0} C}{R} \sec \frac{A_{0} C}{R}
$$

Eq.5 in this paper minus Eq.4 in literature[1] leaves

$$
\Delta h_{A B}=\left[\frac{\left(B_{0} C\right)^{2}}{2 R}-h_{C B}-b\right]\left[\cos \frac{B_{0} C}{R} \sec \frac{A_{0} C}{R}-1\right]
$$

Based on Eq. 6 in this paper, because of $b\left\langle 3\right.$ meters, $A_{0} C\left\langle 200\right.$ meters , $\left.B_{0} C\right\rangle 0$ meter , $h_{C B}\langle 3$ meters and $R=6370000$ meters in practice, we can get $\left|\Delta h_{A B}\right|\left\langle 2.9 \times 10^{-6}\right.$ millimeters. So, we can completely neglect the difference between Eq.4 in literature[1] and Eq. 6 in this paper. 


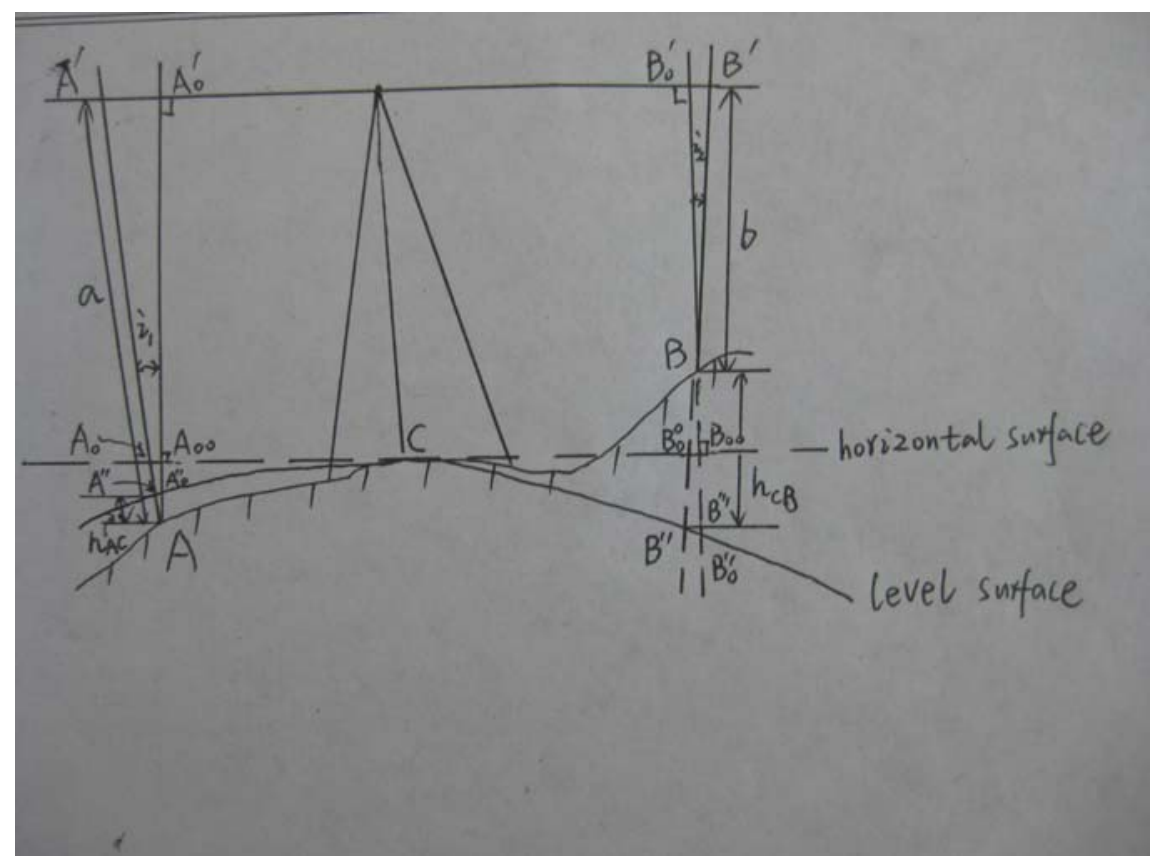

Fig.3 Selecting the horizontal surface and the level surface through point $\mathrm{C}$ to analyse (There $\mathrm{C}$ lies between $\mathrm{A}$ and $\mathrm{B}$ )

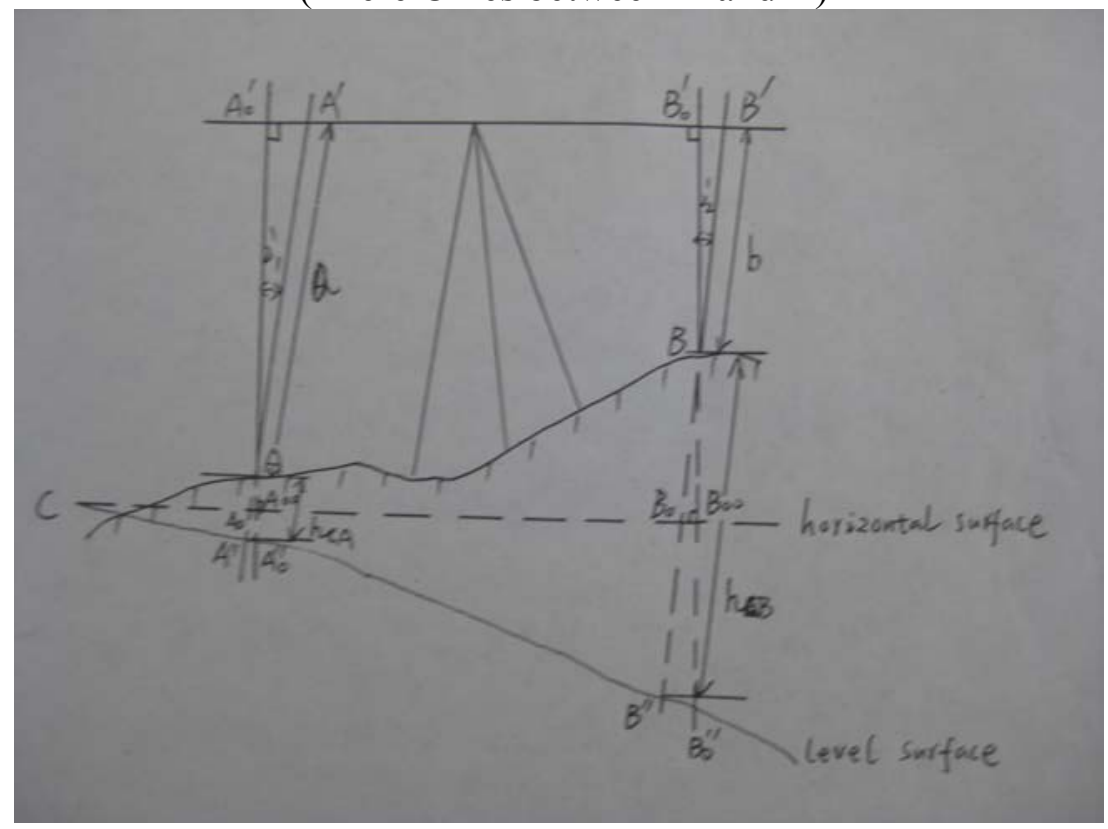

Fig.4 Selecting the horizontal surface and the level surface through point $\mathrm{C}$ to analyse (There $\mathrm{C}$ lies beyond $\mathrm{A}$ and $\mathrm{B}$, and close to $\mathrm{A}$.)

According to the same method in Fig.3 in this paper,we can get $h_{A B}$ and $\Delta h_{A B}$ in Fig.4 and Fig.5 which are the same as Eq.5 and Eq.6.Finally,we found their results are the same as that of Fig. 3 in this paper. 


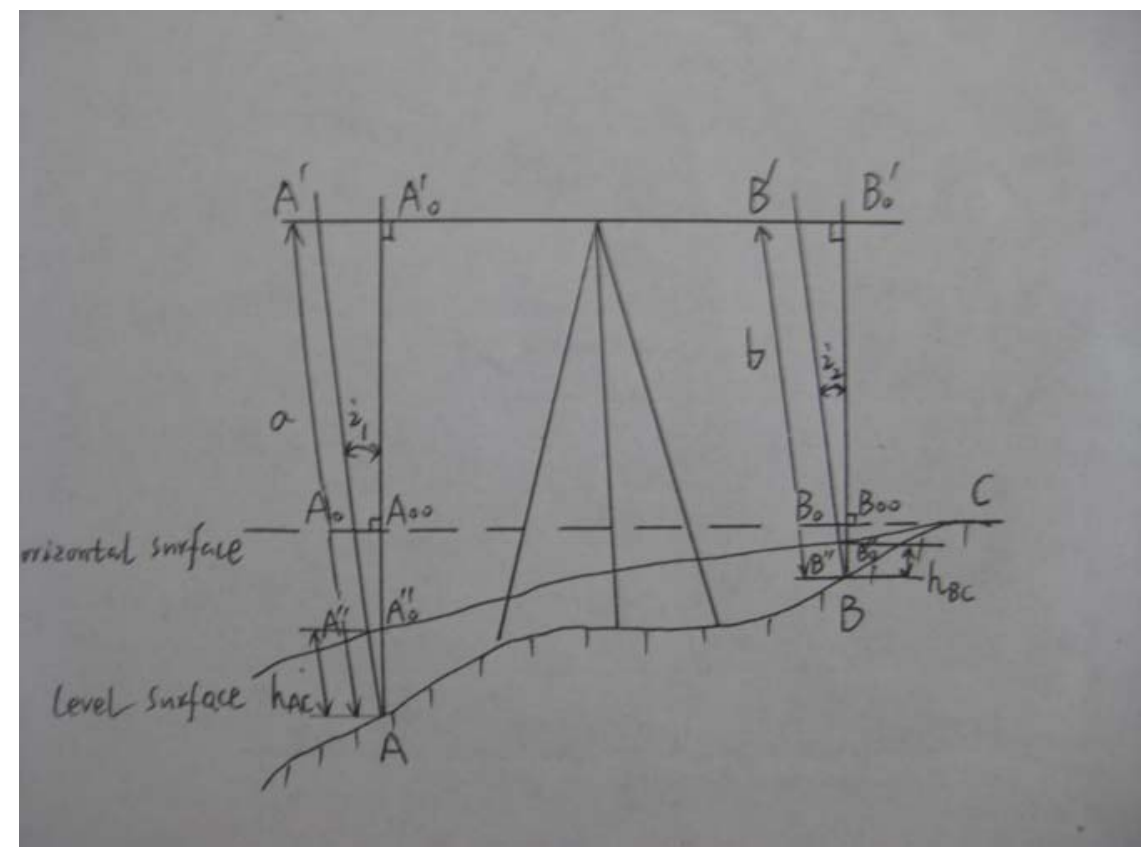

Fig.5 Selecting the horizontal surface and the level surface through point $C$ to analyse (There $\mathrm{C}$ lies beyond $\mathrm{A}$ and $\mathrm{B}$, and close to $\mathrm{B}$.)

\section{Conclusions}

1) This paper has deduced strict formulas of level surveying, but formulas are complex .

2) Formulas in literature [1] are not strict enough, but they can completely replace homologous formulas of this paper in practice.

3) This paper supports and perfects literature[1].

\section{References}

[1] Yonghe Deng. Exploring The Principle of Level Surveying [J].Applied Mechanics and Materials, 170-173(2012):p2962-2965

[2] Hui Qin, Xin Wu.Civil Engineering Surveying (The Third Edition) [M].Shanghai:Tongji University Press,2008(In Chinese).

[3] Nong Wang, Jingjun Guo.Modern General Surveying(The Second Edition)[M].Beijing: Tsinghua University Press,2009(In Chinese).

[4] Zhengfeng Pan, Zhengyao Yang,Xiaojun Cheng,etal. Principles and Method of Digital Mapping[M].Wuhan:Wuhan University Press,2004(In Chinese).

[5] Xiaolie Gu, Feng Bao, Xiaojun Cheng.Surveying (The Third Edition)[M].Shanghai:Tongji University Press,2006(In Chinese).

[6] Yonghe Deng. Improvement of Leveling Principle in College Teaching Materials about Surveying[J]. Railway Investigation and Surveying,3(2011):p7-8(In Chinese). 